\title{
Medium access control in vehicular ad hoc networks
}

\author{
Supeng Leng ${ }^{1,2}$, Huirong $\mathrm{Fu}^{1 *}$, Qing Wang ${ }^{2}$ and Yan Zhang ${ }^{3}$ \\ 1 Department of Computer Science and Engineering, Oakland University, Rochester, MI 48309, U.S.A. \\ 2 School of Communication \& Information Engineering, University of Electronic Science and Technology of China (UESTC), China \\ 3 Simula Research Laboratory, Norway
}

\begin{abstract}
The distinguishing properties of Vehicular Ad hoc wireless Networks (VANETs) strongly challenge the design of Medium Access Control (MAC) protocols, which are responsible for the medium access coordination among active vehicles, as well as the accommodation of both driving safety applications and non-safety applications. In this paper, we focus on a comprehensive survey of VANET MAC schemes by integrating various related issues and challenges. Our analysis not only deepens the understanding of MAC techniques in VANETs but also presents the key ideas and potential directions for future research in this area. In order to significantly improve the communication performance of VANETs, more research efforts on MAC techniques must be made for optimizing multichannel coordination and allocation approaches, enhancing the Quality of Service (QoS) capability, and combating the hidden terminal problem, broadcast storm problem and even ACK (acknowledgment) explosion problem. Copyright @ 2009 John Wiley \& Sons, Ltd.
\end{abstract}

KEYWORDS

vehicular ad hoc wireless network; MAC; multichannel; reliable broadcast; QoS; safety message

${ }^{*}$ Correspondence

Huirong Fu, Department of Computer Science and Engineering, Oakland University, Rochester, MI 48309, U.S.A.

E-mail: fu@oakland.edu

\section{INTRODUCTION}

Vehicular communications are becoming the cornerstone in the future vehicle equipment. A large number of interesting and desired applications of Intelligent Transportation Systems (ITS) are stimulating the development of Vehicular Ad hoc Networks (VANETs), in which network nodes are road-side infrastructure units (RSUs) and vehicle equipped with on-board units (OBUs) for information processing and wireless communication. Since OBUs can act as both end users and wireless routers, Inter-Vehicle Communication (IVC) and Vehicle-to-Roadside Communication (VRC) will be possible over single hop or multiple hops.

The emerging wireless vehicular communication technologies are intended to improve safety and comfort of transportation system. Applications in Wireless Access in Vehicular Environments (WAVE) fall into safety applications and non-safety applications. Safety applications, providing drivers information about critical situations in advance, have strict requirements on communication reliability and delay. On the other hand, non-safety applications, such as on board internet access, electronic map update, and driving through payment, meant for improving driving comfort and the efficiency of transportation system are bandwidth-sensitive.
In order to provide diverse applications in VANETs, a reliable and efficient Medium Access Control (MAC) protocol is required. However, the following characteristics of VANETs challenge the design of an ideal MAC protocol.

- Reliable and fast dissemination for safety messages: Safety information such as safety/emergency messages must be delivered to each host nearby without significant delay. The Vehicle Infrastructure Integration (VII) initiative in the United States proposes that any accident information should be communicated through VANET within $0.5 \mathrm{~s}$ to all equipped vehicles in a safe range [1]. Any delayed or lost safety message could result in loss of life.

- Point-to-point and point-to-multipoint communication: Point-to-point communication is used when a message is intended for a specific receiver. On the other hand, point-to-multipoint communication is used when a message is intended for multiple receivers. Particularly, broadcast is widely used to accommodate point-to-multipoint communication in VANETs, such as safety message and WAVE Service Announcement (WSA) message delivery.

- Relevance-based delivery: Most driving related announcements usually affect the behavior of other 
specific drivers in the vicinity. For instance, the brake messages are very important for the cars behind less than a few hundred meters on the same lane. However, the going straight message of a vehicle is valuable for almost all vehicles near an intersection without traffic lights. Therefore, if message delivery strategies take into account the relevance of information with respect to the potential receivers, the redundant transmission can be largely reduced.

- Highly dynamic topology: A VANET is characterized by rapid topology change due to the constantly moving and changing vehicles, which results in short link connection duration and variable channel quality. Highly dynamic topology makes the traditional MANET (Mobile Ad hoc NETwork) routing protocols incapable for a VANET. However, because a vehicle's movement is constrained by the road, the further movement of a vehicle is predictable with the aid of available location information and electronic map, such as Global Position System (GPS) navigation devices.

- Variable host density: The rapid vehicle movement leads the host density in a network to vary frequently. In the region close to a congested intersection, more than 100 vehicles may contend the shared wireless channel. In this case, the transmission of safety messages becomes a tough task. Contrarily, on a low traffic road, the WAVE channels may always keep in idle state.

- Opportunistic multi-hop data exchange: The centerbased communication provided by a RSU cannot be always available in a VANET, where most applications in VANET rely on multi-hop intervehicle communication. Nevertheless, if only a small number of WAVE transceivers are available in a region, network fragmentation becomes a common problem. In this condition, vehicles can only perform opportunistic data exchange with limited communication duration.

It is obvious that VANETs are fundamentally different from ordinary MANETs [2]. As a consequence, only few MAC protocols designed for MANETs are capable of working in VANETs, where an ideal MAC protocol should be able to utilize the channel resource efficiently, avoid channel access contention smartly, disseminate safety message timely, deliver differentiated traffics properly, and self-configure system parameters adaptively.

In spite of the tremendous ongoing academic and industrial research efforts on VANETs, the proposed MAC solutions allow VANETs to work well only in some limited scenarios. In this paper, we provide a comprehensive overview of techniques and research issues for transmission capability improvement and QoS provisioning in MAC protocols for VANETs. These techniques represent the $\mathrm{R} \& \mathrm{D}$ efforts both in the research community and the ITS industry. Specifically, we firstly summarize the operations of IEEE 802.11p and IEEE 1609.4, which provide the Dedicated Short Range Communication (DSRC) [3] technology platform. Secondly, we introduce the various multichannel coordination and allocation approaches to improve the capacity of MAC protocols. Thirdly, we address the open issues and possible solutions for QoS MAC design, which aims at reducing dissemination latency for delaysensitive traffics and offering differentiated transmissions in service channels. Finally, since the unstable properties of shared WAVE channels strongly affect the reliability of broadcast, we discuss the mechanisms in MAC layer to help broadcast approaches to reduce interference, improve the efficiency of acknowledgment, and suppress redundant packet forwarding. Although the design of MAC protocols also includes other techniques, such as framing, synchronization, and security management, this paper does not address these topics due to page limitation.

The rest of this paper is organized as follows. The active international standards on DSRC MAC are presented in Section 2. Section 3 reviews the current MAC protocols that can be used in VANETs. We also propose the framework of enhanced diversification of IEEE 1609.4 MAC, as well as the further research work. Section 4 gives a short survey on the QoS provision strategies in MAC layer for both safety and non-safety related applications. Section 5 presents the approaches to providing reliable and efficient MAC broadcast in VANETs. Finally, Section 6 concludes this paper.

\section{STANDARDIZATION ACTIVITIES ON DSRC MAC}

Recently, the governments, standardization bodies, automobile manufacturers, and academia are working together to develop VANET-based communication technologies and standards through several initiatives [4 ]. Standardization is at the core of the current and future success of the MAC layer design for VANETs.

DSRC provides high-speed communication between the vehicles and roadside equipments, which is working in the $5.9 \mathrm{GHz}$ band in U.S. and $5.8 \mathrm{GHz}$ band in Japan and Europe. Currently, the organizations CEN/TC278 of Europe and ISO/TC204 of Japan have published its DSRC standards. In North America, ASTM and IEEE committees develop a serial of standards for the implementation of DSRC.

Figure 1 illustrates the evolution of the standards for DSRC system, as well as their scopes. Since the FCC allocated the $5.9 \mathrm{GHz}$ band for DSRC in October 1999, the ASTM has been working on the standards for DSRC devices. ASTM E2213-02 issued in October 2002 describes a MAC layer and physical layer (PHY) specification based on the IEEE 802.11a. IEEE adopted the open DSRC standard ASTM E 2213-03 [5] in 2003. Then, an IEEE working group is investigating a new PHY/MAC amendment of the 802.11 standard designed for VANETs, which is referred as IEEE 802.11p [6]. Moreover, IEEE P1556 aims at defining essential protective mechanisms for DSRC applications and communications technology. In 


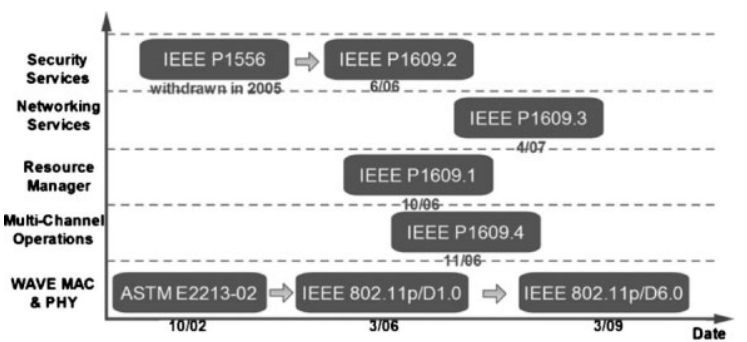

Figure 1. Standards for DSRC system.

December 2005, this standard was withdrawn, and then, it has been superseded by the IEEE 1609 standard family.

The overall DSRC communication stack between the link layer and applications can be standardized by the IEEE 1609 working group. Channelization and the upper layers of the network stack are defined in IEEE P1609.4 and IEEE P1609.3, respectively. IEEE P1609.1 defines the Resource Manager which uses the network stack for communications. IEEE P1609.2 specifies security services for the WAVE networking stack and applications.

Among the current DSRC standards, IEEE 802.11p and IEEE 1609.4 draft [7] define the details of PHY and MAC specifications. IEEE 802.11p employs the Orthogonal Frequency Division Multiplexing (OFDM) technique on the PHY, which can provide up to $27 \mathrm{Mb} / \mathrm{s}$ data rate with $10 \mathrm{MHz}$ bandwidth and 300-1000 m communication distance. The IEEE 802.11p MAC layer inherits the Enhanced Distributed Coordinator Function (EDCF) in IEEE 802.11e [8], which applies Access Category (AC), Arbitration Inter-Frame Space (AIFS), and Transmission Opportunity (TXOP) to support priority in traffic system. Different from Basic Service Set (BSS) in traditional 802.11 standards, the WAVE Basic Service Set (WBSS) introduced in $802.11 \mathrm{p}$ does not require MAC layer authentication and association prior to data transmission within a Distribution Set [6].

As shown in Figure 2, the overall bandwidth defined by IEEE $802.11 \mathrm{p}$ is divided into seven frequency channels. The $\mathrm{CH} 178$ is the Control Channel $(\mathrm{CCH})$, which is used as a public channel for providing safety relevant applications and exchanging control messages among vehicles. The other six channels are Service Channels (SCHs) for supporting the non-safety service applications.

The IEEE 1609.4 standard draft defines a multichannel wireless radio operation mode, including the interleaving operation of $\mathrm{CCH}$ and $\mathrm{SCH}$, priority access parameters, and other characteristics of MAC and PHYs. In order to coordinate the channel access to the $\mathrm{CCH}$ and multiple SCHs, IEEE P1609.4 developed a globally synchronized channel coordination scheme based on the Coordinated Universal Time (UTC). As illustrated in Figure 2, the channel access time is divided into synchronization intervals with a fixed length of $100 \mathrm{~ms}$, consisting of a $\mathrm{CCH}$ interval and an $\mathrm{SCH}$ interval, $50 \mathrm{~ms}$ each. According to the coordination scheme, all devices must tune to $\mathrm{CCH}$ during $\mathrm{CCH}$ phases to exchange safety messages and control messages. During SCH phases, devices can optionally switch to SCHs to perform non-safety applications. This scheme allows the transmission of safety messages and non-safety application on different channels during different intervals, without missing important messages on $\mathrm{CCH}[9]$.

The IEEE 1609.4 standard draft together with IEEE $802.11 p$ provides the framework of multichannel media access control for VANETs. However, as a contention based mechanism, the current WAVE MAC is intuitively questionable on its ability of supporting either delay sensitive applications or throughput sensitive applications.

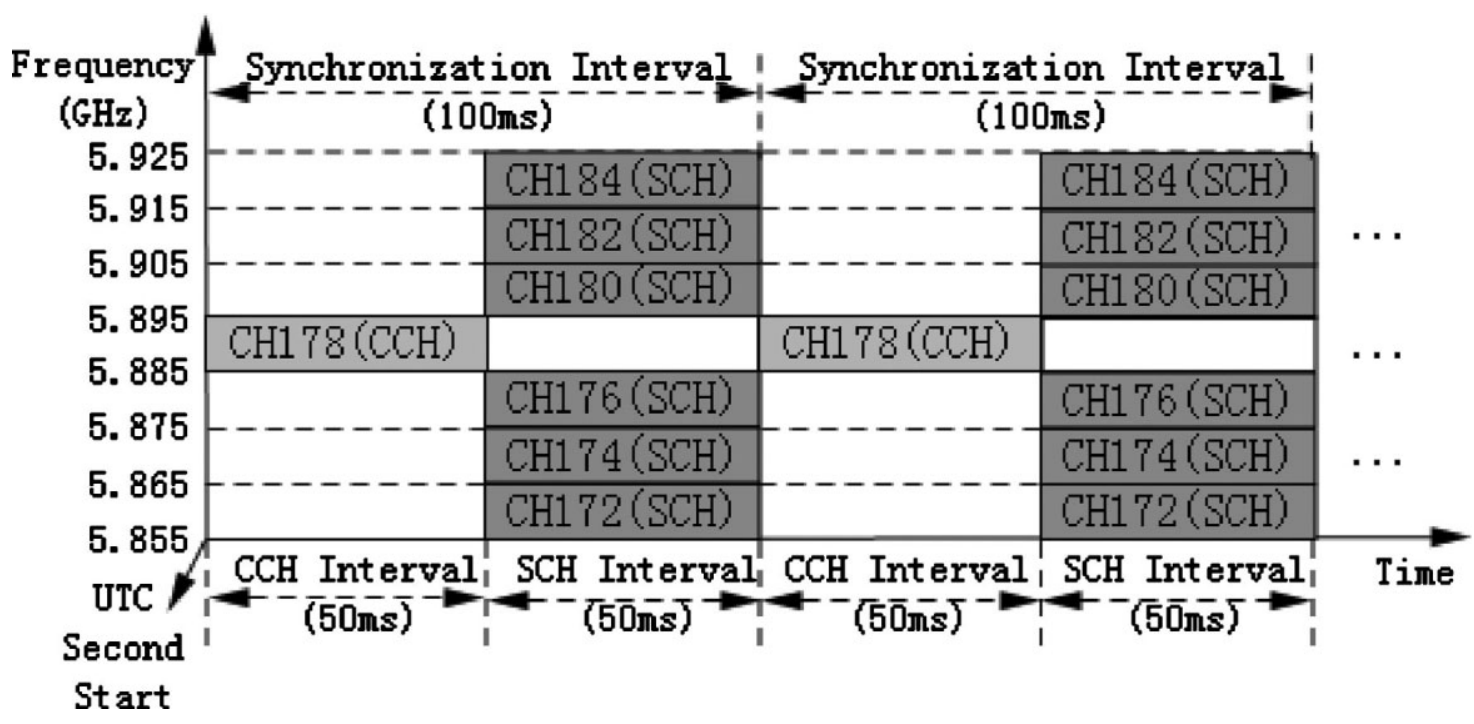

Figure 2. Frequency channel layout of $5.9 \mathrm{GHz}$ WAVE system. 


\section{MULTIPLE CHANNEL ACCESS CONTROL}

Since the maximum throughput of a single channel scheme is limited by the bandwidth of that channel, using more channels appropriately can potentially increase the throughput and reduce the delay. Data transmission on different channels does not interfere with each other, so that multiple transmissions can take place in the neighborhood simultaneously. Therefore, the WAVE standard adopts a multichannel concept for the design of MAC protocols.

The multichannel MAC protocols are essential to not only ensure the reliable transmission of safety messages with low latency, but also provide the maximal throughput for non-safety applications in a distributed manner. In particular, vehicles rely on the channel coordinative strategy (defined by the MAC protocol) to cooperate the medium access behaviors between the $\mathrm{CCH}$ and the $\mathrm{SCH}$. In addition, the MAC protocol should have a smart approach to allocating multiple channel resource for non-safety applications. In this section, we first discuss the existing channel coordination schemes and multichannel allocation approaches. Then, we propose the framework of an IEEE 1609.4 MAC enhancement scheme.

\subsection{Channel coordination}

In the literature [10], Mo et al. classified multichannel MAC protocols for wireless networks into four categories based on the coordination principles. In a Dedicated Control Channel protocol, every device has two radios. One radio is tuned to the channel dedicated to control messages and the other radio tuned to any other channel for data transmission. In Common Hopping approaches, every device requires only one transceiver. Devices not exchanging data hop through all channels synchronously. A pair of devices stop hopping upon making an agreement for transmission. In the Split Phase approaches, devices use a single radio. Time is divided into an alternating sequence of control and data exchange phases. The last category is Parallel Rendezvous protocols, in which multiple devices can use different channels in parallel to exchange control information and make new agreements.

In the future, it is possible that both OBU and RSU equip transceivers with multiple radios. Although the multiple radio MAC protocols (Dedicated Control Channel and Parallel Rendezvous protocols) can take advantage of the seven DSRC channels to enhance the efficiency of message transmission, the multiple transceivers increase the system complexity as well as cost. When DSRC is initially deployed, however, it is envisioned that WAVE devices have only a single radio for accessing or sensing single channel at any instant, which is considered as the major form of DSRC transmission. Among these multichannel MAC protocols, the Common Hopping protocols are suffering from non-negligible hopping time penalty and tight synchronization requirement. Consequently, most
VANET researchers prefer easier implemented Split Phase approaches, including the MAC protocol described by IEEE 1609.4.

Considering the coordinative multichannel access techniques outlined in IEEE 1609.4, we can find one limitation that the ratio between $\mathrm{CCH}$ interval and $\mathrm{SCH}$ interval has a fixed value. In a congested vehicular traffic condition, the limited length of $\mathrm{CCH}$ is unable to provide sufficient bandwidth to deliver large amount of safety messages and control messages, meanwhile, the hosts cannot make enough reservation for data transmission during SCH interval, so that the network performance will be degraded significantly. On the other hand, if the device density is sparse, the occasional transmission on the $\mathrm{CCH}$ channel will waste some of $\mathrm{CCH}$ interval. In this case, however, some large bandwidth consuming applications, such as FTP, video download and map update, cannot obtain enough bandwidth resource on the SCHs.

The Multichannel MAC (MMAC) protocol [11] enables wireless hosts to switch channels dynamically. At the beginning of each beacon interval, i.e., ATIM window, every host listens on a common channel to negotiate data channels. After the ATIM window, hosts tune to their agreed channel and exchange messages for the rest of the beacon interval. Simulation results show that MMAC successfully exploits multiple channels to improve total network throughput over IEEE 802.11 single-channel and the Dynamic Channel Assignment (DCA) multiple channel MAC protocol [12]. On the other hand, the ATIM window is a major overhead in MMAC. Hosts cannot exchange data packets during the ATIM window, even if the exchange of ATIM packets has been completed. The fixed size of ATIM window in MMAC obstructs the further enhancement of transmission efficiency.

Chen et al. [13] proposed a more efficient Multichannel Access Protocol (MAP) using single transceiver in IEEE 802.11 Wireless Local Area Networks (WLANs). All MAP compatible mobile stations contend channel resource in a dedicated channel during a periodical Contention Reservation Interval (CRI), and then transmit data packets over different channels in the Contention-Free Interval (CFI) by a Channel Scheduling Algorithm (CSA). Since the CRI is fixed and the interval of CFI is depending on the scheduling of contention results of CRI, a whole transmission interval is variable. The MAP protocol achieves an obviously higher throughput than conventional IEEE 802.11 MAC protocol and DCA scheme. However, the CSA algorithm and the variable interval of CFI mechanism are only feasible in a central access control manner.

The literature [14] proposed a MAC protocol to support the multichannel operation for DSRC. Their focus is on the challenge of providing potentially high bandwidth for non-safety applications provided by roadside infrastructure, without compromising safety communication occurring in a separate channel. Their architecture tries to solve the channel coordination problem in the presence of a RSU, and compliments with existing ad hoc approaches when no 
RSU is available. However, each device must equip with different protocols in the MAC layer and network layer for both ad hoc mode and infrastructure mode, which increases the complexity of mode switching management. In addition, their work did not address the coordination of control messages, safety messages, and non-safety messages in $\mathrm{ad}$ hoc mode.

Moreover, Jiang et al. proposed the Peercast model in Reference [15], where the device on $\mathrm{SCH}$ regularly switches to $\mathrm{CCH}$ and listens for a few packets from its neighbors. If no safety message is heard, the device will return back to $\mathrm{SCH}$. Although their scheme can improve the throughput of $\mathrm{SCH}$, some safety message may be lost because devices in the network may not stay on the $\mathrm{CCH}$ simultaneously.

\subsection{Multichannel allocation}

In a Split Phase DSRC MAC protocol, the Multichannel allocation approach aims at achieving high throughput on service channels. Although only a few researchers have discussed the multichannel resource allocation for VANET, there has been substantial interest on channel assignment schemes for multi-hop ad hoc networks [12,1618]. However, the channel assignment issue has been shown to be NP-complete graph coloring problem [19], and thus computationally intractable.

Consider that a common non-safety application usually has one service provider and multiple service users, the sender-based channel allocation schemes are applied in VANETs, in which the sender always decides a data channel according to its own selection criterion to exchange data with a receiver. The receiver may either conditionally or unconditionally accept the decision [20]. For a receiver unconditional acceptance scheme, the channel contention strategy is required on the selected data channel to prevent collision at the receiver side. Contrarily, if the receiver conditionally accepts the decision made by the sender, the decision can be rejected upon the violation of its own selection criterion. This may involve several rounds of negotiations (two-way handshakes) between the sender and receiver. However, for the point-to-multipoint communications in a VANET, it is difficult to negotiate between the service provider and multiple users. In this case, each service provider needs to collect enough information of neighbors for optimal channel selection in an unconditional way.

According to the literature [20], three criteria are commonly used in channel selection for multi-hop ad hoc networks, namely, idle state, traffic load, and random assignment schemes. In the idle state schemes $[13,16,18,21,22]$, the channel that will become idle at the earliest is selected. The traffic load schemes [10,23] select the channel with the lightest traffic load. Although the use of the above two criteria will incur the overhead on information collection, they are widely used in service channel allocation in VANETs. On the contrary, a random assignment Scheme [24] has poor performance due to random channel selection.
It is still an open issue to design efficient channel allocation schemes for the non-safety applications in VANETs. In Reference [25], the proposed Vehicular MESH Network (VMESH) MAC protocol is compliant with the multichannel operation defined in IEEE 1609.4. Instead of contention-based access, VMESH devices apply a distributed beaconing scheme and a reservation based channel access scheme on $\mathrm{SCH}$ to improve the channel utilization. Upon receiving the beacon from a service provider, the device initiates its reservation request based on the traffic load and the heard SCH occupancy information of neighbors. Assigned with specific beacon slots, the RSUs can efficiently allocate the channel resource within its range. The separated Beacon Period and Safety Period in $\mathrm{CCH}$ interval eliminate the interference between the control messages and the high priority safety messages. VMESH outperforms typical WAVE MAC schemes in terms of system throughput. However, this MAC protocol is not suitable for the highly dynamic and fully distributed network environment.

Zhang et al. [26] proposed a cluster-based multichannel communications scheme, which integrates the clustering with MAC protocols. The elected cluster-head $(\mathrm{CH})$ vehicle functions as the coordinator to collect and deliver the real time safety messages within its own cluster, and forward the consolidated safety messages to the neighboring $\mathrm{CHs}$. The $\mathrm{CH}$ vehicle also controls channel-assignments for non-realtime traffics among cluster-member vehicles. Their scheme uses the contention-free TDMA MAC within a cluster and the IEEE 802.11 MAC among $\mathrm{CH}$ vehicles. Each clustermember vehicle uses one transceiver to exchange the safety messages with its $\mathrm{CH}$ vehicle, and uses another transceiver to communicate with other cluster members over the data channel assigned by its $\mathrm{CH}$ vehicle. According to the simulation results, their scheme can significantly improve the throughputs of vehicle data communications while guaranteeing the real-time delivery of safety messages. The drawback of this protocol is the complicated cluster management strategy and the hardware cost of dual transceivers in each vehicle.

In the literature [27], Xie et al. proposed a multi-channel MAC protocol for dense VANET with directional antennas, which can increase the spatial reuse of wireless channels. Channel allocation is conducted by RTS/CTS with a beam table that indicates the current state of beams in all seven DSRC channels. The unblocked beams can be used as long as the wireless nodes are in unblocked state. However, most beams will be blocked because of the sensed busy channels. The transmission efficiency can be further improved if their approach is adaptable for vehicular speed.

The literature [28] introduced the cognitive radio technology to the design of WAVE systems. The authors presented a Cognitive MAC Protocol for VANET (CMV). Their focus is on integrating the aspects of spectrum access in terms of both long-term and short-term time scales. In order to achieve long-term spectrum access, each WAVE device probes the channel condition every $\mathrm{CCH}$ and $\mathrm{SCH}$ interval, and maintains a spectrum table for the sensed 
spectrum condition. A WAVE device applies a simple voting process to choose the spectrum which can provide good performance and cause little interference in the neighborhood. For short-term spectrum access, the device uses wideband spectrum pooling technique together with wide-RTS, CTS to reflect the spectral condition near the actual receiver, and choose a qualified $\mathrm{SCH}$. Nevertheless, the computational complexity and convergence speed of CMV should be evaluated carefully.

In order to be qualified in the complicated conditions of VANETs, an ideal multichannel allocation scheme should have the on-demand channel assignment principle for only active devices. More importantly, an efficient channel allocation scheme should also associated with the vehicle mobility [29], link quality, spectrum resource, transmission power, and interference level. The link available time can be predicted with the aid of localization technique and electronic maps. When the distance between vehicles becomes large, the increase of transmission power is helpful for maintaining the network connectivity, but causes more interference on the assigned channel in the proximity. One possible solution for transmission power adaptation is performing local estimation of vehicles density [30] as well as traffic load.

\subsection{IEEE 1609.4 enhancement}

Recently, IEEE 1609.4 is considered to be a default multichannel MAC standard for VANETs. Although IEEE 1609.4 defines the general framework for multichannel management, this standard draft does not describe the detail of MAC coordination scheme. Nevertheless, WAVE devices have to content both $\mathrm{CCH}$ and SCHs for each packet. This is improper and inefficient for a VANET with the characteristics of highly dynamic topology change and traffic priority differentiation.

It is obvious that this standard draft needs to improve the coordinative management of $\mathrm{CCH}$ and SCHs. In
Reference [31], we proposed a Variable CCH Interval (VCI) multichannel MAC scheme to accommodate real-time and throughput-sensitive services, by using a multichannel coordination mechanism and variable intervals of $\mathrm{CCH}$ and $\mathrm{SCH}$.

The multichannel coordination mechanism provides contention-free SCHs that rely on the channel contention and reservation through $\mathrm{CCH}$. As shown in Figure 3, upon beginning a $\mathrm{CCH}$ interval, service providers content $\mathrm{CCH}$ to broadcast the WSA messages. The winner selects the service channel, which accommodates the least number of service transmissions, to send its service packets in the $\mathrm{SCH}$ interval.

We also proposed a variable $\mathrm{CCH}$ interval approach, which adjusts the ratio between the $\mathrm{CCH}$ interval and the SCH interval according to the network condition. As shown in Figure 3, upon beginning the $\mathrm{CCH}$ interval, the RSU broadcasts a VCI message, containing the value of the $\mathrm{CCH}$ interval $T_{\mathrm{cch}}$, to the hosts under its radio coverage range. The optimal value of $T_{\text {cch }}$ must ensure the successful transmission of safety messages as well as WSA messages under the coverage range of the RSU. On the other hand, the interval of $\mathrm{CCH}$ should be limited in order to improve the channel utilization of SCHs. Therefore, $T_{\text {cch }}$ is proportional to the total number of service providers but inversely proportional to the bandwidth of $\mathrm{CCH}$.

Simulations are conducted to compare the performance of three MAC schemes, i.e., the original WAVE MAC, the Fixed CCH Interval (FCI) MAC scheme with multichannel coordination mechanism, and the VCI MAC scheme. The only difference between the last two MAC schemes is that the VCI MAC can adjust the interval of $\mathrm{CCH}$, instead of a fixed $50 \mathrm{~ms} \mathrm{CCH}$ interval used in the FCI MAC. Figure 4 shows the throughput of SCHs in terms of the length of service messages in the three MAC schemes. It can be seen that the VCI MAC outperforms the other two schemes with either small or large packet length. This is because that the VCI MAC has larger SCH interval for service packet transmission. We can also find that the original

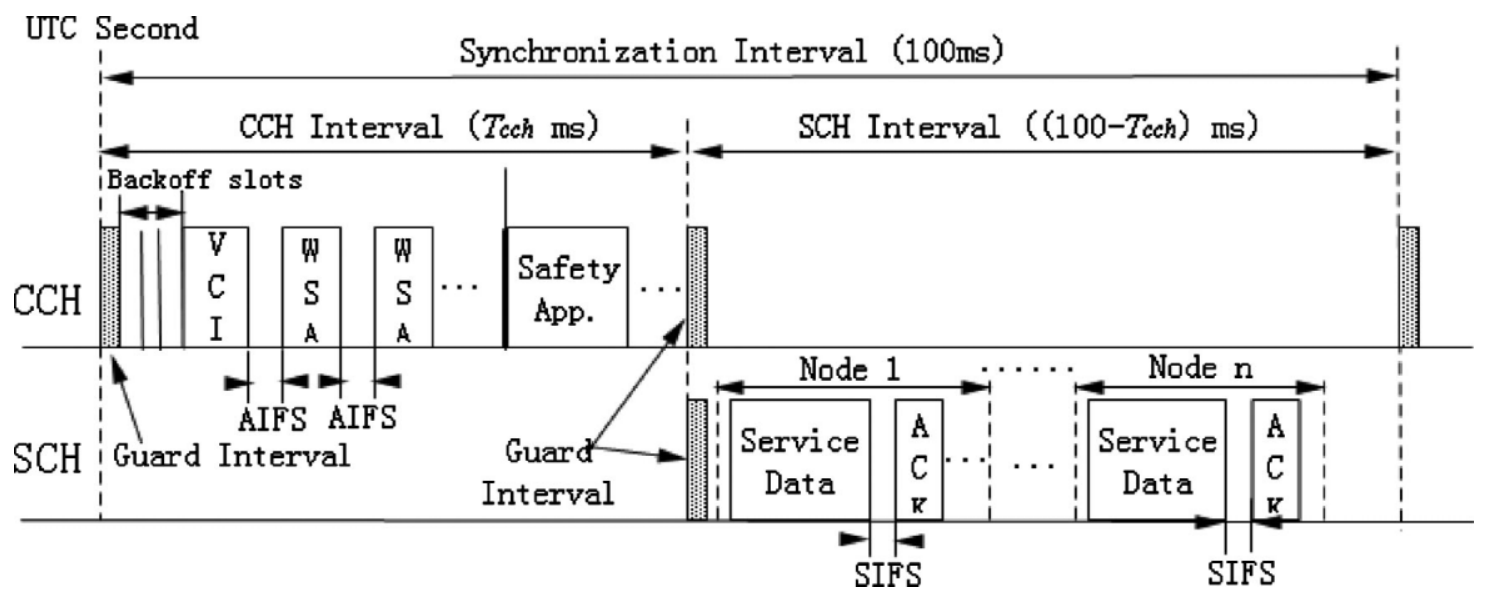

Figure 3. The $\mathrm{VCl}$ multichannel MAC scheme. 


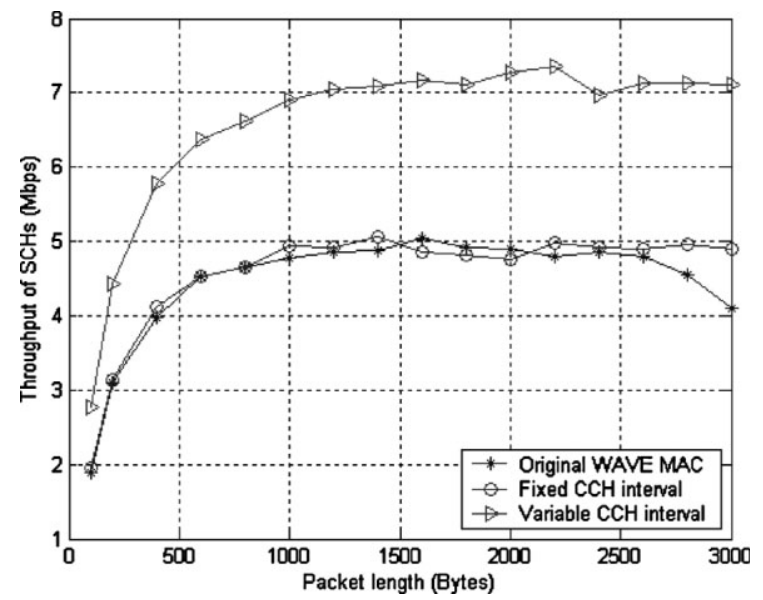

Figure 4. Throughput of $\mathrm{SCH}$ s under different MAC schemes.

WAVE MAC and the FCI MAC have similar throughput in most conditions. However, when the packet length grows to more than 2500 bytes, the throughput in the original WAVE MAC decreases significantly because of serious contentions. Contrarily, with the aid of the multichannel coordination mechanism, both the FCI MAC and the VCI MAC are able to keep stable throughput in a contention free channel environment.

In the VCI MAC scheme, the multichannel coordination mechanism can provide contention free channel access in service channels. The variable $\mathrm{CCH}$ interval scheme is able to balance the tradeoff between safety messages delivery in $\mathrm{CCH}$ and the efficient transmission in SCHs. However, the current version of VCI cannot dynamically adapt the $\mathrm{CCH}$ interval according to the traffic load of $\mathrm{CCH}$ and SCHs. To calculate the optimal interval, we need to study the relationship between control overhead and data transmission workload for various applications. Another issue is that the $\mathrm{CCH}$ intervals announced by different RSUs may be variable. It is challenging to design the synchronization mechanism for the channel intervals in a distributed multi-hop wireless environment, which can be used to determine the $\mathrm{CH}$ interval of the hosts located in the overlapping coverage range of two and more RSUs.

Table I summarizes the main features of the abovementioned multichannel MAC approaches. We also include in the table whether the MAC solutions are able to adjust $\mathrm{CCH}$ interval dynamically or utilize cognitive radio technique and smart/directional antenna, since the communication performance of VANETs will be significantly improved by using these advanced technologies.

\section{MAC LAYER QOS PROVISION}

Because of the shared wireless channel with a CSMA/CA medium access scheme (such as IEEE 802.11p) and multi-hop communication between distant vehicles, the limited bandwidth in a VANET is further decreased by poor channel utilization. In such a complicated WAVE environment, it is a challenging task to provide delaysensitive delivery for safety applications, while satisfying various QoS requirements of non-safety applications. In fact, QoS support need to be implemented at several layers, which involve system architecture, QoS routing protocol and QoS MAC scheme. Due to the limited length, this section only studies medium access mechanisms supporting QoS control, which focus on delivery latency reduction in control channel and various types of traffic offering in service channels.

\subsection{Latency reduction}

The traditional ad hoc network packet-forwarding approaches may be only applicable for relatively large delay-tolerant data applications, such as in-vehicle Internet services. Nevertheless, these approaches are not adequate for low-latency driving safety applications. Driver's reaction time to stimulation like brake lights can be of the order of $0.7 \mathrm{~s}$ and even higher [32]. Thus, if the single-hop transmission delay comes in larger than $0.5 \mathrm{~s}$, the driver may realize danger prior to the emergency alert system.

Table I. Classification and comparison of multichannel MAC for DSRC.

\begin{tabular}{|c|c|c|c|c|c|c|c|}
\hline Solutions & Channel coordination & $\begin{array}{c}\text { Radio } \\
\text { interface }\end{array}$ & $\begin{array}{l}802.11 p \\
\text { based }\end{array}$ & $\begin{array}{l}\text { IEEE1609.4 } \\
\text { compatible }\end{array}$ & $\begin{array}{l}\text { Adjustable } \\
\mathrm{CCH} \text { interval }\end{array}$ & $\begin{array}{l}\text { Cognitive } \\
\text { radio }\end{array}$ & $\begin{array}{l}\text { Smart } \\
\text { antenna }\end{array}$ \\
\hline MMAC [11] & $\begin{array}{l}\text { Split phase with ATIM } \\
\text { window }\end{array}$ & 1 & No & No & $\mathrm{N} / \mathrm{A}$ & No & No \\
\hline MAP [13] & Split phase & 1 & No & Partially & N/A & No & No \\
\hline $\begin{array}{l}\text { Multichannel coordinated } \\
\text { mode [14] }\end{array}$ & Split phase & 1 & Partially & No & N/A & No & No \\
\hline Peercast [15] & Split phase & 1 & Yes & Partially & Yes & No & No \\
\hline VMESH [25] & Split phase & 1 & Yes & Yes & No & No & No \\
\hline $\begin{array}{l}\text { Cluster-based multichannel } \\
\text { Scheme [26] }\end{array}$ & Dedicated control channel & 2 & Partially & No & $\mathrm{N} / \mathrm{A}$ & No & No \\
\hline VMMAC [27] & Split phase & 1 & Yes & No & N/A & No & Yes \\
\hline CMV [28] & Split phase & 1 & Yes & Yes & No & Yes & No \\
\hline VCl [31] & Split phase & 1 & Yes & Yes & Yes & No & Yes \\
\hline
\end{tabular}


On the other hand, the number of active hosts has large impact on network connectivity and the likelihood of channel blocking. In a VANET scenario with dense vehicles such as a congested intersection, hundreds of vehicles attempt to send safety messages, exchange control messages, and broadcast WSA in the control channel. In this case, the most crucial bottleneck is the limited bandwidth, which leads to serious channel contention, and long latency for the dissemination of safety messages.

In Reference [33], Lee et al. proposed a wireless token ring MAC protocol (WTRP) for platoon vehicle communication, in which all participating vehicles form a group and drive cooperatively. This protocol is suitable for slow moving scenarios. The literature [34] proposed a time slot-based Reservation ALOHA (R-ALOHA) MAC protocol for inter-vehicle communication. The Reliable R-ALOHA (RR-ALOHA) protocol [35] is the extension version for the purpose of achieving dynamic time slot allocation, where each vehicle needs to select for itself one basic channel (BCH), i.e., one time slot periodically repeated in successive packets. Furthermore, each vehicle has a thorough view of the transmissions in a two-hop neighborhood to overcome the hidden terminal problem. However, the above two TDMA-based MAC protocols request tight synchronization and cannot differentiate the priorities of emergency messages and common applications.

Reference [36] reviews the existing variants of the 802.11 DCF to support QoS. The 802.11e working group has been chartered to enhance the QoS capability. The EDCF of IEEE 802.11e [8] gives multiple priority value for each type of traffic flows. Voice or audio traffic that needs low latency can be guaranteed to get smaller media access latency than the other traffics. However, how to deploy EDCF to deliver short safety messages in VANET is still an open topic.

In EDCA, service differentiation and thus the delay sensitive traffic class can be supported by setting up a multiple channel access parameters, such as increasing priority value or reducing backoff parameters for highpriority traffic. However, IEEE $802.11 \mathrm{e}$ does not take into account of link state, dynamic topology issues and the impact of multi-hop, which are very common in a VANET. Moreover, when numerous messages with equal priority are delivered on a heavy traffic load channel, for example, emergency alert dissemination in a dense vehicle environment, the collision probability is very high. It is necessary to improve the performance of priority access supporting mechanism, such as backoff strategy, which is able to suppress the contention adaptively according to the instant link state. One solution [37] is combining the current backoff strategy of EDCA with dynamic $p$ persistence algorithm, which determines the transmission probability in each contention window slot, based on the collision time in the control channel.

In addition, the lifetime of safety messages needs to be concerned in the design of VANET MAC protocols. If a safety application updates a message every $0.5 \mathrm{~s}$, but the delivery latency is more than $0.5 \mathrm{~s}$ after it is created, then the message received may be always stale. Hence, the delay-tolerant delivery must match the requirement of the messages' lifetime. This also ensures the availability of the message for a new vehicle entering the area after the initial broadcast.

\subsection{QoS support in service channels}

As ITS system tends to carry delay-sensitive data, audio/video traffic and internet browsing, the accommodation of different non-safety applications with certain levels of QoS becomes a must feature. Non-safety applications increase the overall comfort of the driver, but consume a large amount of channel resources. Compared with safety messages, the non-safety traffic has lower channel access priority and is transmitted in an opportunistic manner. While the existing QoS supporting approaches are adapted for multi-hop wireless networks (especially wireless mesh networks), research along this direction for non-safety applications in VANET is still in the initial stage.

DSRC adopts the EDCA [38,39] of IEEE 802.11e provides differential access to the wireless medium by assigning eight priority classes, referred to as Access Categories (AC). EDCA uses a different set of access parameters for each AC. Service differentiation and thus the delay requirement of time-bounded traffic class can be supported by setting up a multiple channel access parameters, such as priority value and backoff window size. To match the structure of the multiple WAVE channels, the priority queuing scheme for the access categories applies one set of queues for the services channels and another set of queues for the control channel, in which the control channel is always given priority over service channels.

It has been studied in Reference [40] that IEEE 802.11e can provide certain QoS support in a single hop WLAN. However, the literature [41] reports that QoS support in a multi-hop VANET using EDCA has weak performance. It is obvious that each traffic flow competes for the channel with all other flows in wireless LANs, however in multihop networks, every flow may have different experience which depends on the network topology and flow pattern. For example, in a multi-hop network, flow $A$ and flow $B$ have the same priority. The former contents the channel with up to other 10 flows, while the latter only competes with flow $A$. In such a circumstance, the packets of flow $B$ has more chance to be delivered than that of flow $A$, and the different data transmission rates result in unfairness.

A contention based VANET MAC scheme needs not only to differentiate multiple traffic classes, but also consider the traffic load of each class. There are several control mechanisms that can be used to improve the design: (1) IFS values (that determines when a host can start to count down its backoff timer upon sensing an idle medium), (2) the minimum and maximum contention windows $(C W m i n$ and $C W$ max: the range in which backoff time value can be randomly selected), (3) the number of retransmission attempts, (4) the backoff scaling factor (that affects the 
increase intensity of the contention window upon collision), (5) the maximal frame size, and (6) the transmission opportunity limit (TXOP: that determines how long a host can transmit once holding the channel).

Reference [36] presents a comprehensive review on the approaches for QoS provisioning in IEEE 802.11-compliant networks. Existing approaches have explored use of one or more of the above mechanisms. Although the above (1), (2), and (6) mechanisms have been defined in the EDCA approach, IEEE 802.11e does not take into account of channel contention level, link quality, host mobility, and the wireless hop number, which are the important factors in medium access control for VANETs.

\section{RELIABLE AND EFFICIENT MAC BROADCAST}

Chen et al. [42] have addressed three categories of data dissemination in VANET, i.e., broadcast/geocast, multicast, and unicast. Broadcast is the major transmission form to offer point-to-multipoint communications in VANETs, especially for the delivery of safety messages. Although broadcast is more efficient than multicast [43] or unicast $[44,45]$, the reliability of broadcast is still affected by the interference from hidden terminals in multihop wireless environment. The wireless link quality may deteriorate due to multipath fading, shadowing, and Doppler shifts caused by the high mobility of vehicles. Moreover, the suppression of redundant broadcast messages brings additional difficulty to the design of an efficient safety message dissemination approach.

Effective broadcasting depends strongly on the interaction between the routing and MAC layers. Intuitively, a broadcast strategy may lead to significantly high delivery latency, as the large number of redundant transmissions at the MAC layer cause contention-induced backoff and Broadcast Storm Problem [46]. Furthermore, using unreliable MAC approaches, it is possible to lose broadcast messages due to interference or transmission errors. Obviously, MAC protocols play an important role in broadcasts in wireless vehicular networks.

In this section, combining the design of MAC approaches, we will address the issues and relevant solutions for broadcast in VANETs, in terms of interference mitigation, acknowledgments issue, and overhead reduction.

\subsection{Interference mitigation}

Since the IEEE 802.11 MAC protocol is usually considered to be a default standard in multi-hop wireless networks, most broadcast schemes are based on the CarrierSense Multiple-Access/Collision-Avoidance (CSMA/CA) technique. However, the RTS/CTS handshake cannot be used to reserve the channel resource for multiple broadcasting receivers. It is very difficult that the transmitter negotiates with every receiver before broadcast. Moreover, the packets of RTS or CTS vary in the range between 14 and 20 bytes [6], which is about $20 \%$ of the average length of a safety message (approximately 100 bytes). In this case, the use of RTS/CTS handshake is costly to reserve channel for safety messages. Nevertheless, without the aid of RTS/CTS handshake, the hidden terminal problem in a multi-hop network may cause large amount of packet collisions, especially in a dense active host environment.

Matsuda et al. [47] proposed a multi-hop broadcast flooding relay protocol that filters forwarded packets taking account of delay, relative distance, and other metrics on slotted Aloha. In References [23], [48] and [49], the authors described token-based protocols that only permit sources holding the token to transmit, so there are no collisions at the MAC layer. But how to avoid token loss and duplicate tokens in highly dynamic vehicular environments are critical issues. Reference [50] develops a MAC protocol using directional antennas and directional handshake upon highway scenario and urban scenario mobility model. The proposed approach allows pairs of terminals with idle beams to connect in the same channel to reduce the hidden terminals. There are some other protocols in the literatures that have been designed for broadcast traffic in vehicular networks, such as References $[1,35,51,52]$. However, these literatures ignore the hidden terminal problem during broadcasting.

On the other hand, the control message based mechanisms for transmission negotiation may fail in channel reservation in a dynamic VANET environment, since the neighboring hosts of the receiver may not be able to hear the handshake such as RTS/CTS. From this viewpoint, among the existing CSMA/CA MAC protocols, only the busy tone aided protocols are able to solve hidden terminal problem effectively. The DBTMA protocol [53] introduces two out-of-band busy tones to indicate the ongoing transmissions, in which one busy tone indicates busy transmitting and the other shows busy receiving. When hearing any busy-tone, the neighboring hosts are prohibited from transmitting. Moreover, in Reference [54], power control is deployed to further increase channel utilization. A sender uses an appropriate power level to transmit packets so as to avoid interrupting on-going packet receptions. In the literature [55], the authors proposed a similar pulsebased MAC scheme to realize strict packet-level priority scheduling for emergency packets in VANETs, where both the transmitter and the receiver send priopulses in the control channel to suppress hidden terminals.

Recently, a lot of research efforts [56-58] have been made to study the effect of large interference range on the multihop network performance. The term large interference range denotes the situation that the interference range of a host is larger than its transmission range. The design of these traditional MAC protocols was usually based on the assumption that the interference range of a mobile host is the same as its transmission range. This ideal assumption may not match the realistic environment. As a consequence, few 


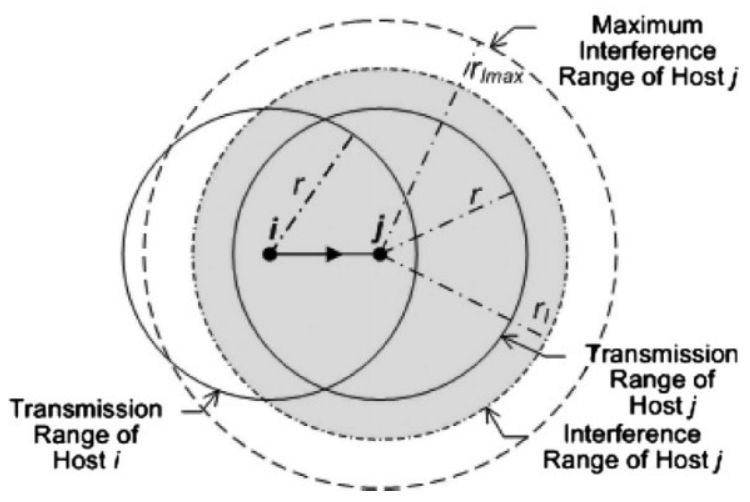

Figure 5. System model for large interference area problem.

MAC protocols are able to achieve expected performance when the large interference area problem is present.

Figure 5 shows the system model for large interference area problem, in which host $i$ intends to transmit data packet to host $j$ with distance $x$. When there is an ongoing communication between hosts $i$ and $j$, other hosts in the neighborhood may transmit packets and become potential interfering hosts. Let $r$ denote the radio transmission range of hosts in a multi-hop wireless network. In the open space environment, if the transmission power of a packet is $P_{\mathrm{t}}$, the received signal power at the distance $x$ is given by Reference [59], that is

$$
P_{\mathrm{r}}(x)=\frac{P_{\mathrm{t}} G_{\mathrm{t}} G_{\mathrm{r}} h_{\mathrm{t}}^{2} h_{\mathrm{r}}^{2}}{x^{k}}
$$

where $G_{\mathrm{t}}$ and $G_{\mathrm{r}}$ are the antenna gains of transmitter and receiver, respectively, $h_{\mathrm{t}}$ and $h_{\mathrm{r}}$ are the height of both antennas, and $k$ reflects how fast the signal decays.

Considering the signal-to-interference ratio (SIR), a signal at a receiver is valid only if its measured SIR is no lower than a threshold $T_{\mathrm{SNR}}$ [58]. Let $P_{i}$ be the power of interference signal at host $j$ with distance of $y$ from the interfering host. Then, host $j$ is able to successfully receive packets from host $i$, if SIR $=P_{r}(x) / P_{i}(y)=(y / x)^{k} \geq T_{\mathrm{SNR}}$. Thus, the interference range of a receiver is defined as $r_{\mathrm{I}}=\sqrt[k]{T_{\mathrm{SNR}}} \cdot x$.

As shown by the shadow area in Figure 5, hence the distance $x$ between $i$ and $j$ is larger than $r\left(T_{\mathrm{SNR}}\right)^{-1 / k}$, the transmission range is smaller than the interference range of the receiver. Since $0 \leq x \leq r$, the maximum interference range is $r_{\text {Imax }}=\sqrt[k]{T_{\mathrm{SNR}}} \cdot r$. In this case, the RTS/CTS handshake alone cannot prevent possible collisions caused by the large interference area problem.

Researchers have proposed several MAC approaches $[56,60,61]$ to reduce the effect of large interference area problem on unicast through multi-hop wireless networks. One major solution is to adjust the power level of RTS/CTS or busy tones so that their transmission ranges are no less than the interference range of hosts. For example, we proposed the power-fixed dual (PFD) and the power-aware dual (PAD) busy-tone schemes in Reference [61], through which the coverage range of busy tones can indicate the maximum interference range $r_{\text {Imax }}$ and the exact interference range $r_{\mathrm{I}}$, respectively.

However, the existing research work has not addressed broadcast mechanism in multi-hop wireless networks with a large interference area. Since busy tones can always stay on to avoid any potential interfering host within the entire transmission duration of a message, the busy tone can be used to protect the reception of broadcasting messages in VANET. Based on calculation, the transmission power of busy tone can be adjusted to make sure that its sensing range equals to the largest interference range of receivers.

It is worth noting that such calculation only considers the transmission power of busy tone for individual host. If multiple receiving hosts transmit the broadcast busy tone simultaneously, this may accumulate the receiving power of busy tone, which will enlarge the blocking area for ceasing the transmission of neighbors. As the result, during the receiving of a broadcast message, the neighboring hosts, which can sense the accumulated busy tone signal but locate outside of the receivers' interference range, have to defer their transmission and waste the channel resource. The future research work can aim at the design of optimal power control mechanism that takes account of the accumulative effect of busy tones launched by multiple transceivers, as well as the aggregate interference from all simultaneous transmissions in the network.

\subsection{Acknowledgement and retransmission}

Reliable communication in wireless networks typically means retransmitting a packet till it is acknowledged by the recipients. This is appropriate for file transfers since even one missing byte renders the entire packet unusable. But the paradigm is not appropriate for safety messages via broadcast. It is not practical for a transmitter to collect ACK packets from every receiver for a broadcast message, since acknowledgments cause ACK explosion problem [62]. If every receiver sends an ACK back to the transmitter, numerous channel contentions and message collisions happen.

On the other hand, the link quality in a VANET changes frequently. It may also elevate the chance of channel collisions because the carrier sense mechanism is affected, particularly in a heavy traffic load network. In this condition, packet loss is a serious problem for safety message dissemination. Without the aid of acknowledgment, the transmitter cannot make sure that the broadcast message reaches the receivers successfully. Furthermore, acknowledgment is one of important failure detection mechanisms for packet transmission that can be used to adjust contention window upon packet collision in WAVE channels.

Consequently, as the detection mechanism of failed broadcast, acknowledgment is widely deployed for safety message dissemination. In order to solve the ACK explosion problem, some approaches $[63,64]$ use the neighboring topology information to designate the hosts that will send 
back ACK. However, this kind of methods cannot ensure the correct reception by all receivers. Alternatively, VANET hosts can detect collisions and congestion by analyzing the sequence numbers of the received packets [65]. Based on the observation of local condition, hosts are able to adjust the contention window size and thus improve performance. But receivers may not hear the short safety messages sent towards them so that the estimation of traffic load condition may not be accurate.

To increase the probability of message reception, another solution is rebroadcasting each message several times. According to the DSRC standard [66], each vehicle broadcasts its status to the neighbors approximately 10 times every second. The main drawback of repetition is the channel resource consumption by excessive redundant messages, especially in a VANET with high vehicle density. Moreover, how to guarantee the reception of a safety message by all receivers is still an open issue.

\subsection{Dissemination overhead control}

The basic dissemination scheme used to realize broadcastbased VANETs is flooding, through which each host forwards every single message it receives. The main problem of flooding mechanism is large amount of superfluous transmissions leading to network congestion, which has been known as the Broadcast Storm Problem [46]. This effect is aggravated with an increasing host density and network size, leading to weak scalability. In such a condition, the limited wireless channel resource is largely absorbed by redundant traffic. Thus, time-critical messages may be prevented from accessing the shared medium.

Current research on optimal broadcasting mechanisms in multi-hop wireless networks has been focusing on minimizing the number of rebroadcasts while keeping high broadcast reliability. According to the types of information required, these broadcast protocols can be grouped into two categories: topology-based and geometry-based.

In topology-based broadcast protocols [67-71], hosts determine whether the received packet is forwarded according to the connectivity information in their neighborhood. Since an optimal broadcast forwarding in ad hoc network is proven to be NP-complete [72], topologybased protocols attempt to approximate the minimal connected cover set. Each host is assumed to know the local connectivity information up to two hops. Based on the topology information, topology-based protocols can generate a small forwarding host set, so that the redundant rebroadcasts can be significantly reduced while maintaining the maximum broadcast reachability. However, the exchange of local topology information under such a topology-based protocol may cause a large amount of overhead, since each host must maintain a long list of the neighboring hosts, especially when the host density in the network is high. In addition, hosts in the network need to take a long time to collect all connectivity information with the other neighboring hosts within two hops $[67,68,72]$, which makes the created forwarding host set incorrect. Hence the topology-based broadcast protocols cannot maintain good performance in a highly dynamic VANET environment.

In contrast, the geometry-based broadcast protocols choose the forwarding hosts according to the geometry location information of direct neighboring hosts. Each host obtains its location information either through low power low cost GPS receivers, or by measuring signal strengths and calculating relative coordinates [73]. The location information is exchanged among direct neighboring hosts via periodical beacons or broadcasting packets. The exchange of location information only consumes a small amount of bandwidth. Compared with topologybased protocols, the geometry-based protocols [46,74-76] usually have less convergence time to obtain the location information, and they give each host less computational load to generate the cover set. Therefore, the geometry-based protocols are more efficient for a VANET network with a high level of host mobility. On the other hand, because of insufficient network topology information, the performance of geometry-based protocols is usually poorer than that of topology-based protocols.

According to the characteristics of VANETs, many researchers developed more sophisticated dissemination schemes for safety messages. They address that the dissemination protocols for vehicular safety applications should preferably be broadcast oriented and they should rely on packet forwarding based on geographic, directional, and other relevant temporal contexts of the source and the destination vehicles.

Collision warning services are addressed in the literature [77], which presents a directional broadcasting protocol using geographical information. The proposed mechanism implements a receiver oriented next forwarder choice. Each receiving host sets up a timer on the basis of its own position and its distance to the destination, according to the qualitative criteria that hosts in better positions will have shorter timers. Upon timer expiration, the receiving host forwards the packet and all the hosts overhearing this transmission simply abort their own. Nevertheless, multiple unwanted transmissions of the same packet may still happen. The literature [50] proposed the TLO (The Last One) broadcast algorithm that uses GPS information to improve the performance of safety alert application. Their approach chooses the last vehicle, i.e., farthest from the place of accident, to rebroadcast emergency message, thus reduce the broadcast storm, as well as the probability of message collision.

Campelli [78] addressed the design of position based routing solutions for the support of safety oriented applications in VANETs, by focusing on the impact of the dynamic TDMA scheme on the routing performances. They proposed a directional broadcast solution which leverages the position based routing paradigm and the trajectory based one. However, high vehicular mobility makes this TDMA MAC coordination in a VANET environment more difficult than that in traditional wireless scenarios. Xu et al. [79] 
discussed a vehicle-to-vehicle location-based broadcast communication protocol, in which each vehicle generates emergency messages at a constant rate. The optimum transmission probability at MAC layer for each message is then identified to reduce the message collision probability.

Geocast performs the transmission of a message to some or all hosts within a geographical area, which naturally match the requirements of many VANET applications with destinations locating in a specified area. Previous research work on geocasting for vehicular networks presented various flooding based schemes. The literature [80] proposed an efficient geocast algorithm for the support of virtual warning signal, whose target is to distribute the information within a defined geocast zone. The proposed scheme adopts a unicast routing to reach the destination area by the selected forwarding host that is closest to the destination. The geocast algorithm needs the topological information of the neighboring hosts, and applies a greedy algorithm for the choice of the next forwarder.

A system of abiding geocast was designed in Reference [81] for disseminating vehicular warning message. Vehicles on a stretch of roads carry safety messages to the vehicles moving in the opposite direction, so as to warn a dangerous situation ahead. Consequently, all relevant vehicles receive the warning before reaching the dangerous area, whereas only a few messages are broadcasted. Furthermore, since the wait time of individual relay vehicles are set dynamically for the next broadcast, the unnecessary broadcasts can be largely saved.

Ibrahim et al. [82] presented a probabilistic Inter-Vehicle Geocast ( $p$-IVG) scheme to address this spatial broadcast storm problem. A $p$-IVG compliant vehicle re-broadcasts frames probabilistically depending upon the traffic density in the vicinity. Using $p$-IVG results in higher reception rates, lower channel contention, and most importantly, faster dissemination of data to distant vehicles than the original geocast approach.

The above approaches can be considered as a starting point to design more elaborate schemes for safety message dissemination. However, theses approaches do not take into account the relevance of the information with respect to the potential receivers. In the literature [83], the relevance of information has been used as a priority metric to schedule channel access. In fact, the use of the information relevance-related delivery can be further extended to reduce dissemination overhead.

According to the literature [84], the current VANET applications can be classified into five communication patterns that have different communication mechanisms and delivery direction. In the patterns of Geobroadcast and Advanced Information Dissemination, most disseminated information only needs to reach the vehicles that would be interested in it. For example, when an accident occurs, only those vehicles in the risk region concern the emergency alarm message. Vehicles in these risk regions constitute a dynamic group, which may changes frequently according as the information content and the receiver's geographic location. However, the constraint parameters

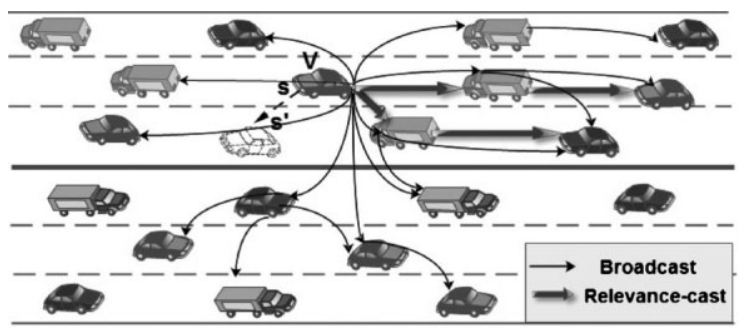

Figure 6. Broadcast versus relevance-cast.

and forwarding forms must be carefully designed in an application specific manner.

The redundant broadcast messages can be reduced significantly upon using the information relevance-related delivery. This new dissemination method is named as relevance-cast, which is different with the ordinary broadcast and geocast. As shown in Figure 6, a vehicle $\mathrm{V}$ intends to change the lane from the place $\mathrm{s}$ to s' on a highway. The relevant safety message should be forwarded to the vehicles driving behind less than a few hundred meters on the current lane and the new lane. However, if broadcasting is applied, the message will be delivered to all the vehicles in the range of two or three wireless hops, and then numerous redundant message forwarding attempts may block the wireless channel in the neighborhood. On the contrary, relevance-cast makes the message reach only a few relevant vehicles, thus the redundant forwarding is suppressed significantly.

One basis of relevance-cast is the differentiation of safety services. According to the types of safety service applications, safety messages can be categorized into different classes. Each class has a specific delivery range and direction. The MAC scheduling approaches based on IEEE 802.11 e provide priority differentiated medium access. Upon receiving a safety message, the receiver decides whether the packet relay is necessary based on the class identity. Meanwhile, the smart antenna technique can be applied to implement the precisely directional forwarding, which can further suppress channel contention and message collisions.

It can be seen that more research efforts should be made to improve the performance of broadcast mechanisms for VANETs. We can apply the following parameters to evaluate the reliability and efficiency of a new broadcast approach.

- Message collision ratio: the average percentage of unsuccessful broadcast attempts which are corrupted by other messages.

- Channel utilization: the channel throughput over the channel bandwidth.

- Blocking area: the reserved area for an on-going transmission, which reflects the channel spatial reuse capability.

- Aggregate throughput: the maximal total throughput of simultaneous transmissions in a network. 
- The control overhead: the average cost of transmission control messages (such as RTS/CTS and ACK) in terms of bps.

- The delivery ratio: the average percentage of reachable hosts for receiving a specific class of messages in a network.

- The number of forwarding hosts: the average number of hosts that are involved in messages forwarding.

- The average end-to-end delay: the average delay of a message transmitted from the source to the destination, including the propagation delay, the transmission delay, and the processing delay in WAVE devices.

\section{CONCLUSION}

There is no doubt that vehicular ad hoc wireless networks will play an important role in the future ITS. Different types of traffic information could be delivered to drivers through vehicle-to-vehicle and vehicle-to-infrastructure communications. However, the distinguishing characteristics of VANETs make the existing MAC protocols for ordinary MANETs unsuitable in a WAVE environment. This paper gives a global review of media access control techniques for VANETs. We summarize recent work in this domain including MAC standardization activities, wireless channel resource management, QoS capability enhancement in MAC layer, and reliable MAC broadcasting strategies. The technologies used for vehicular networks are still not mature and will probably not be implemented in the immediate future. As shown in this paper, there are still many issues that must be addressed before a practical VANET can be deployed. It is anticipated that the current IEEE 802.11p and IEEE 1609.4 MAC specifications will need to be suitably altered to meet the transmission requirements of DSRC applications.

\section{ACKNOWLEDGEMENTS}

This work was partly supported by the National Natural Science Foundation of China under Grant No. 60802024, the Research Fund for the Doctoral Program of Higher Education of China under Grant No. 200806141014, the Research Fund of National Communication Laboratory, UESTC, China, and the U.S. National Science Foundation under Grants No. 0716527 and No. 0736877, Michigan Space Grant Consortium Research Seed Grant, and Oakland University Faculty Research Fellowship (FRF). Any opinions, findings, and conclusions or recommendations expressed in this material are those of the authors and do not necessarily reflect the views of the National Science Foundation.

\section{REFERENCES}

1. Xu Q, Mak T, Ko J, Sengupta R. Vehicle-to-vehicle safety messaging in DSRC. In 1st ACM International
Workshop on Vehicular Ad hoc Networks (VANET) 2004; 19-28.

2. Blum J, Eskandarian A, Hoffman L. Challenges of intervehicle ad hoc networks. IEEE Transactions on Intelligent Transportation Systems 2004; 4(5): 347-351.

3. Armstrong L. Dedicated short-range communications project. URL: leearmstrong.com/DSRC/ DSRCHomeset.htm

4. Moustafa H, Zhang Y. Vehicular Networks: Techniques, Standards and Applications, Auerbach Publications, Taylor\&Francis Group: USA, 2008.

5. ASTM E2213-03, Standard specification for telecommunications and information exchange between roadside and vehicle systems $-5 \mathrm{GHz}$ band dedicated short range communications (DSRC) medium access control (MAC) and physical layer (PHY) specifications, ASTM International, August 2003.

6. IEEE P802.11p/D6.0, Draft Amendment for Wireless Access in Vehicular Environments (WAVE), March, 2009.

7. IEEE 1609.4. IEEE Trial-Use Standard for Wireless Access in Vehicular Environments (WAVE) - Multichannel Operation, November, 2006.

8. IEEE Std 802.11-2007: Wireless LAN Medium Access Control (MAC) and Physical Layer (PHY) Specification, 2007.

9. Yunpeng Zang, Stibor L, Walke B, Reumerman HJ, Barroso A. A novel MAC protocol for throughput sensitive applications in vehicular environments. In IEEE Vehicular Technology Conference (VTC) 2007; 2580-2584.

10. Mo J, So HSW, Walrand J. Comparison of multichannel MAC protocols. IEEE Transactions on Mobile Computing 2008; 7(1): 50-65.

11. So J, Nitin V. Multichannel MAC for ad hoc networks: handling multichannel hidden terminals using a single transceiver. ACM International Symposium on Mobile Ad hoc Networking and Computing (MobiHoc), May 2004; 222-233.

12. Tang J, Xue G, Zhang W. Interference-aware topology control and QoS routing in multichannel wireless mesh networks. In Workshop on Mobile and Ad Hoc Networking and Computing (MobiHOC) 2005; 68-77.

13. Chen J, Sheu S, Yang C. A new multichannel access protocol for IEEE 802.11 ad hoc wireless LANs. In 14th IEEE Proceedings on Personal, Indoor and Mobile Radio Communications (PIMRC) 2003; 2291-2296.

14. Mak TK, Laberteaux KP, Sengupta R. A multichannel VANET providing concurrent safety and commercial services. In 2nd ACM International Workshop on Vehicular Ad hoc Networks 2005; 1-9.

15. Jiang D, Taliwal, Meier V, Holfelder A, Herrtwich W. Design of $5.9 \mathrm{GHz}$ DSRC-based vehicular safety 
communication. IEEE Wireless Communications 2006; 13(5): 36-43.

16. Jain N, Das S, Nasipuri A. A multichannel CSMA MAC protocol with receiver-based channel selection for multihop wireless networks. In 10th International Conference on Computer Communications and Networks 2001; 432-439.

17. Raniwala A, Chiueh T. Architecture and algorithms for an IEEE 802.11-based multichannel wireless mesh network. In 24th Annual Joint Conference of the IEEE Computer and Communications Societies (INFOCOM) 2005; 2223-2234.

18. Wu SL, Lin CY, Tseng YC, Sheu JP. A new multichannel MAC protocol with on-demand channel assignment for multi-hop mobile ad hoc networks. In International Symposium on Parallel Architectures, Algorithms and Networks (ISPAN) 2000; 232-237.

19. Bertossi A, Bonuccelli M. Code assignments for hidden terminal interference avoidance in multi-hop packet radio networks. IEEE/ACM Transaction on Networks 1995; 3(4): 441-449.

20. Shou-Chih L, Chia-Wei T. A novel multichannel MAC protocol for wireless ad hoc networks. In IEEE Vehicular Technology Conference (VTC) Spring, 2007; 46-50.

21. Chen JH, Chen Y. AMNP: ad hoc multichannel negotiation protocol for multi-hop mobile wireless networks. In In IEEE International Conference on Communication (ICC) 2004; 3607-3612.

22. Koubaa H. Fairness-enhanced multiple control channels MAC for ad hoc networks. In IEEE Vehicular Technology Conference (VTC) 2005; 1504-1508.

23. Lee D, Attias R, Puri A, Sengupta R, Tripakis S, Varaiya P. A wireless token ring protocol for intelligent transportation systems. In IEEE Intelligent Transportation System Conference 2001; 1152-1157.

24. Chang CY, Sun HC, Hsieh CC. MCDA: an efficient multichannel MAC protocol for 802.11 wireless LAN with directional antenna. In 19th International Conference on Advanced Information Networking and Applications (AINA) 2005; 64-67.

25. Zang YP, Walke L, Reumerman B, Barroso HJ. Towards broadband vehicular ad-hoc networks-the vehicular mesh network (VMESH) MAC protocol. In IEEE Wireless Communications and Networking Conference (WCNC) 2007; 417-422.

26. Zhang X, Su H, Chen HH. Cluster-based multichannel communications protocols in vehicle ad-hoc networks. IEEE Wireless Communications Magazine 2006; 5(13): 44-51.

27. Xu X, Benxiong H, Shaoshi Y, Tiejun L. Adaptive multi-channel MAC protocol for dense VANET with directional antennas. In Consumer Communications and Networking Conference 2009; 1-5.
28. Seung-eun C, Chong-kwon K. A cognitive MAC for VANET based on the WAVE systems. In 11th International Conference on Advanced Communication Technology (ICACT 2009) 2009; 41-46.

29. Mohimani GH, Ashtiani F, Javanmard A, Hamdi M. Mobility modeling, spatial traffic distribution, and probability of connectivity for sparse and dense vehicular ad hoc networks. IEEE Transactions on Vehicular Technology 2009; 4(58): 1998-2007.

30. Artimy MM, Robertson W, Phillips WJ. Assignment of dynamic transmission range based on estimation of vehicle density. In Second International Workshop on Vehicular Ad Hoc Networks 2005; 40-48.

31. Wang Q, Leng S, Fu H, Zhang Y. A novel multichannel MAC scheme for vehicular ad hoc networks. In 1st International Conference on Wireless Access in Vehicular Environments 2008.

32. Olson P. Perception-response time to unexpected roadway hazards. Human Factors 1986; 28(1): 91-96.

33. Lee D, Attias R, Puri A, Sengupta R, Tripakis S, Varaiya P. A wireless token ring protocol for ad-hoc networks. In IEEE Aerospace Conference 2002; 1219-1228.

34. Verdone R. Multi-hop R-Aloha for inter-vehicle communication at millimeter waves. IEEE Transaction on Vehicular Technology 1997; 46(4): 992-1005.

35. Borgonovo F, Capone A, Cesana M, Fratta L. ADHOC MAC: a new MAC architecture for ad hoc networks providing efficient and reliable point-to-point and broadcast service. Wireless Networks 2004; 4(10): 359366.

36. Kim H, Hou J, Hu C, Ge Y. QoS provisioning in IEEE 802.11-compliant networks: past, present, and future. Computer Networks Journal 2007; 8(51): 19221941.

37. Mao J, Mao Y, Leng S, Bai X. A simple adaptive optimization scheme forIEEE 802.11 with differentiated channel access. IEEE Communications Letters 2009; 13(5): 297-299.

38. Deng J, Chang RS. A priority scheme for IEEE 802.11 DCF access method. IEICE Transactions on Communications 1999; 1(82): 96-102.

39. Mangold S, Choi S, Klein O, Hiertz G. IEEE 802.11e wireless LAN for quality of service. In European Wireless Conference 2002; 32-39.

40. Ni Q. Performance analysis and enhancements for IEEE 802.1le wireless networks. IEEE Networks 2005; 4(19): 21-27.

41. Niu ZY, Yao WB, Ni Q, Song YH. Study on QoS Support in 802.1 le-based multi-hop vehicular wireless ad hoc networks. In 2007 IEEE International Conference on Networking, Sensing and Control 2007; 705-710.

42. Wai C, Guha RK, Taek JK, Lee J, Hsu IY. A survey and challenges in routing and data dissemination in vehicular 
ad-hoc networks. In IEEE International Conference on Vehicular Electronics and Safety 2008; 328333.

43. Kihl M, Sichitiu M, Ekeroth T, Rozenberg M. Reliable geographical multicast routing in vehicular ad-hoc networks. In 5th international conference on Wired/Wireless Internet Communications 2007; 315325.

44. Lo S-C, Lu W-K. Design of data forwarding strategies in vehicular ad hoc networks. In IEEE Vehicular Technology Conference (VTC) 2009; 1-5.

45. Ferreiro-Lage JA, Gestoso CP, Rubinos O, Agelet FA. Analysis of unicast routing protocols for VANETs. In $5 t h$ International Conference on Networking and Services 2009; 518-521.

46. Ni S, Tseng Y, Chen Y, Sheu J. The broadcast storm problem in a mobile ad hoc network. In ACM/IEEE International Conference on Mobile Computing and Networking (MOBICOM) 1999; 151-162.

47. Matsuda S, Koike H, Okada H. Vehicular information broadcasting relay (VIBROR) protocol for intervehicle-communications. In IEEE Vehicular Technology Conference (VTC) Fall, 2000; 2005-2010.

48. Sun Q, Garcia-Molina H. Using ad-hoc inter-vehicle networks for regional alerts. Technical Report, Stanford University 2004.

49. Zhang J, Liu K-H, Shen X. A novel overlay token ring protocol for inter-vehicle communication. In IEEE International Conference on Communications 2008; 4904-4909.

50. Suriyapaibonwattana K, Pomavalai C. An effective safety alert broadcast algorithm for VANET. In International Symposium on Communications and Information Technologies 2008; 247-250.

51. Torrent-Moreno M, Jiang D, Hartenstein H. Broadcast reception rates and effects of priority access in 802.11-based vehicular ad-hoc networks. In 1st ACM International workshop on Vehicular ad hoc networks, 2004; 10-18.

52. Yadumurthy R, Chimalakonda A, Sadashivaiah M, Makanaboyina R. Reliable MAC broadcast protocol in directional and omnidirectional transmissions for vehicular ad hoc networks. In 2nd ACM international Workshop on Vehicular ad hoc networks (VANET) 2005; 10-19.

53. Haas ZJ, Deng J. Dual Busy Tone Multiple Access (DBTMA)-a multiple access control scheme for ad hoc networks. IEEE Transactions on Communications 2002; 50(6): 975-984.

54. Wu SL, Lin CY, Tseng YC, Sheu JL. Intelligent medium access for mobile ad hoc networks with busy tones and power control. IEEE Journal on Select Areas in Communications 2000; 9(18): 1647-1657.
55. Peng J, Cheng L. A distributed MAC scheme for emergency message dissemination in vehicular ad hoc networks. IEEE Transactions on Vehicular Technology 2007; 6(56): 3300-3308.

56. Xu K, Gerla M, Bae S. Effectiveness of RTS/CTS handshake in IEEE 802.11 based ad hoc networks. Ad Hoc Networks 2003; 1(1): 107-123.

57. Ye F, Yi S, Sikdar B. Improving spatial reuse of IEEE 802.11 based ad hoc networks. In IEEE GLOBECOM 2003; 1013-1017.

58. Ray S, Starobinski D, Carruthers JB. Performance of wireless networks with hidden hosts: a queuing-theoretic analysis. Elsevier Computer Communications 2005; 10(28): 1179-1192.

59. Rappaport T. Wireless Communications: Principles and Practice. Prentice Hall: London, UK, 1996.

60. Cesana M, Maniezzo D, Bergamo P, Gerla M. Interference aware (IA) MAC: an enhancement to IEEE $802.11 \mathrm{~b}$ DCF. In IEEE Vehicular Technology Conference (VTC) Fall 2003; 2799-2803.

61. Leng S, Zhang Y, Chen HH, Xiang J, Guizani M. Powerfixed and power-aware MAC protocols for multi-hop wireless networks with large interference area. IEEE Transactions on Vehicular Technology 2009; 58(6): 2966-2976.

62. Ni S-Y, Tseng Y-C, Chen Y-S, Sheu J-P. The broadcast storm problem in a mobile ad hoc network. In ACM/IEEE International Conference on Mobile Computing and Networking (MOBICOM) 1999; 151-162.

63. Tang K, Gerla M. MAC reliable broadcast in adhoc networks. In Military Communications Conference (MILCOM) 2001; 28-31.

64. Sun M-T, Huang L, Arora A, Lai T-H. Reliable MAC layer multicast in IEEE 802.11 wireless networks. In International Conference on Parallel Processing 2002; 527-536.

65. Balon N, Guo J. Increasing broadcast reliability in vehicular ad hoc networks. In Proceedings of the Third International Workshop on Vehicular Ad Hoc Networks 2006; 104-1105.

66. Vehicle safety communications project task 3 final report: identify intelligent vehicle safety applications enabled by DSRC, Vehicle Safety Communications Consortium, 2005.

67. Peng W, Lu X. On the reduction of broadcast redundancy in mobile ad hoc networks. In Workshop on Mobile and Ad Hoc Networking and Computing (MobiHOC) 2000; 129-130.

68. Jie W, Wei L. On reducing broadcast redundancy in ad hoc wireless networks. IEEE Transaction on Mobile Computing 2002; 2(1): 111-122.

69. Sivakumar R, Sinha P, Bharghavan V. CEDAR: a coreextraction distributed ad hoc routing algorithm. IEEE 
Journal on Selected Areas in Communications 1999; 8 : 1454-1465.

70. Lim H, Kim C. Multicast tree construction and flooding in wireless ad hoc networks. In Proceedings of the ACM International Workshop on Modeling, Analysis and Simulation of Wireless and Mobile Systems (MSWIM) 2000; 122-140.

71. Songmin K, Younghwan J, Sangbin L, Eunsook L, Sunshin A. Efficient geocast utilizing topology information database. In IEEE 8th International Conference on Computer and Information Technology Workshops 2008; 210-215.

72. Lim H, Kim C. Flooding in wireless ad hoc networks. Computer Communications 2001; 3(24): 353-363.

73. Capkun S, Hamdi M, Hubaux JP. GPS-free positioning in mobile ad-hoc networks. Hawaii International Conference on System Sciences 2001; 157-167.

74. Tseng Y, Ni S, Shih E. Adaptive approaches to relieving broadcast storms in a wireless multi-hop mobile ad hoc network. IEEE Transaction on Computers 2003; 5(52): 545-557.

75. Sun M, Feng W, Lai T. Location aided broadcast in wireless ad hoc networks. In IEEE Global Telecommunications Conference (GLOBECOM) 2001; 2842-2846.

76. Leng S, Zhang L, Yu LW, Tan CH. An efficient broadcast relay scheme for MANETs. Computer Communications 2005; 28(5): 467-476.

77. Biswas S, Tatchikou R, Dion F. Vehicle-to-vehicle wireless communication protocols for enhancing highway traffic safety. IEEE Communication Magazine 2006; 1(44): 74-82.

78. Campelli L, Cesana M, Fracchia R. Directional broadcast forwarding of alarm messages in VANETs. In 4th Annual Conference on Wireless on Demand Network Systems and Services (WONS'07) 2007; 72-79.

79. Xu Q, Sengupta R, Jiang D. Design and analysis of highway safety communication protocol in $5.9 \mathrm{GHz}$ dedicated short range communication spectrum. In IEEE Vehicular Technology Conference (VTC) Spring, 2003; 2451-2455.

80. Maihofer C, Eberhardt R. Geocast in Vehicular environments: caching and transmission range control for improved efficiency. IEEE Intelligent Vehicles Symposium, 2004; 951-956.

81. Yu Q, Heijenk G. Abiding geocast for warning message dissemination in vehicular ad hoc networks. In IEEE International Conference on Communications Workshops 2008; 400-404.

82. Ibrahim K, Weigle MC, Abuelela M. p-IVG: probabilistic inter-vehicle geocast for dense vehicular networks. In IEEE Vehicular Technology Conference (VTC) 2009; $1-5$.
83. Kosch T, Adler CJ, Eichler S, Schroth C, Strassberger M. The scalability problem of vehicular ad hoc networks and how to solve it. IEEE Wireless Communications 2006; 5(13): 22-28.

84. Schoch E, Kargl F, Weber M. Communication patterns in VANETs. IEEE Communications Magazine 2008; 46(11): 119-125.

\section{AUTHORS' BIOGRAPHIES}

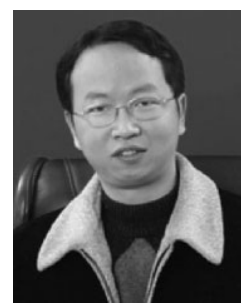

Supeng Leng is an Associate Professor in the School of Communication \& Information Engineering, University of Electronic Science and Technology of China (UESTC). He received his Ph.D. degree from Nanyang Technological University (NTU), Singapore in 2005. He has experience as a R\&D engineer in the field of computer communications, and as a Research Fellow in the Network Technology Research Center, NTU. He is an Editorial Board Member of International Journal of Ultra Wideband Communications and Systems (Inderscience). His research focuses on ad hoc/sensor networks, wireless mesh networks, UWB networks, 4G wireless networks, and cognitive radio networks.

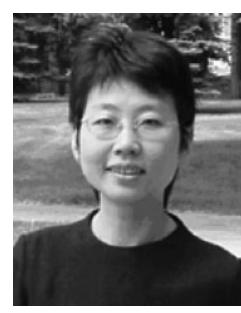

Huirong Fu is an Associate Professor in the Department of Computer Science \& Engineering, Oakland University. She joined Oakland University as an Assistant Professor in 2005. Previously, she has been working as an Assistant Professor at North Dakota State University for 3 years, and as a Post-doctoral Research Fellow at Rice University for more than 2 years. As a lead Professor and the principal investigator in several projects funded by the NSF, $\mathrm{Dr} F u$ has been actively conducting research in the area of information security. Her primary research interests are in information assurance and security, networks, internet data centers, and multimedia system and services.

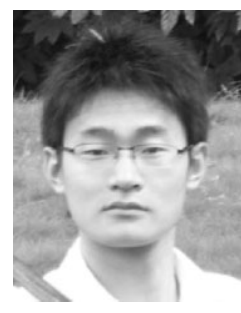

Qing Wang received his B.Eng. degree from University of Electronic Science and Technology of China (UESTC), and is currently working toward his M.Eng. degree in the School of Communication \& Information Engineering, UESTC. His research interests include wireless ad hoc networks and wireless sensor networks. 


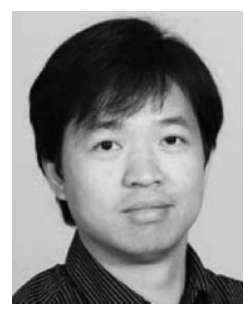

Yan Zhang received his Ph.D. degree from Nanyang Technological University, Singapore. From August 2006, he is working with Simula Research Laboratory, Norway. He is currently serving as the Book Series Editor for the book series on "Wireless Networks and Mobile Communications" (Auerbach Publications, Taylor and Francis

Group). He serves as Program Co-Chair for IWCMC 2009, Program Co-Chair for BROADNETS 2009, Symposium Co-Chair for ChinaCom 2008, Industrial Co-Chair for MobiHoc 2008, Program Co-Chair for UIC-08, Program Vice Co-Chair for IEEE ISM 2007, Publicity Co-Chair for UIC-07, Publication Chair for IEEE ISWCS 2007, Program Co-Chair for IEEE PCAC'07. His research interests include resource, mobility, spectrum, and energy management in wireless networks. 